\title{
Ser verde ou ser sustentável? Uma revisão bibliométrica sobre cadeias de suprimentos verdes e sustentáveis
}

\section{Karina Fernanda da Silva* e Ricardo Silveira Martins}

Universidade Federal de Minas Gerais. Faculdade de Ciências Econômicas. Departamento de Ciências Administrativas. Av. Pres. Antônio Carlos, 6.627. Pampulha. Belo Horizonte-MG, Brasil (CEP 31270-901). E-mail: kafe26@gmail.com.

Resumo. Este estudo teve como objetivos analisar o estado da arte de cadeias de suprimentos verdes e sustentáveis no período de 1982 a 2019, identificando as principais publicações e identificando as diferenças entre elas e as lacunas. A análise de dados dos 244 artigos foi realizada usando os indicadores da análise bibliométrica. Os resultados demonstraram que houve evolução no número de publicações referentes aos temas mostrando que se tratam de temas atuais e relevantes. Nesse período, o país que mais publicou foi a Índia e as principais fontes de publicações foram o Journal of Cleaner Production e Journal of Production Economics. Os resultados também constataram que não basta ter uma cadeia de suprimentos verde para se atingir a sustentabilidade. É preciso ter cadeias de suprimentos sustentáveis e, embora os resultados demonstrem grandes esforços dedicados nesta busca, diversas lacunas e oportunidades ainda existem.

Palavras-chave: Cadeia de suprimentos; Cadeia de suprimentos verde; Cadeia de suprimentos sustentável.

\begin{abstract}
To be green or to be sustainable? A bibliometric review on green and sustainable supply chains. This study aimed to analyze the state of the art of green and sustainable supply chains from 1982 to 2019, identifying the main publications and identifying the differences between them and the gaps. Data analysis of the 244 articles was performed using the indicators of bibliometric analysis. The results showed that there was an evolution in the number of publications referring to the themes, showing that they are current and relevant themes. During this period, the country that published the most was India and the main sources of publications were the Journal of Cleaner Production and the Journal of Production Economics. The results also found that having a green supply chain is not enough to achieve sustainability. It is necessary to have sustainable supply
\end{abstract}

Recebido

$15 / 10 / 2021$

Aceito

03/01/2022

Disponível on line

$31 / 01 / 2022$

Publicado

30/04/2022

Acesso aberto

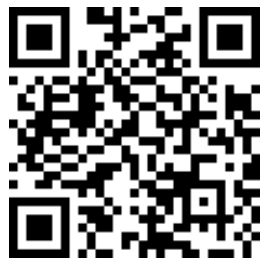

ORCID

(D) 0000-0001-6108-0292

Karina Fernanda da

Silva

D) 0000-0001-9717-3896

Ricardo Silveira

Martins

ISSN 2359-1412/RBGAS-2021-0134/2022/9/21/2/21

Rev. Bras. Gest. Amb. Sustent.

http://revista.ecogestaobrasil.net 
chains and, although the results show great efforts dedicated to this search, several gaps and opportunities still exist.

Keywords: Supply chain; Green supply chain; Sustainable supply chain.

\section{Introdução}

As formas tradicionais de governar não têm conseguido gerenciar inúmeros aspectos da realidade pública. Frente a crescente globalização e industrialização há exigências iminentes para logística verde e sustentável (Ren et al., 2020).

Os motivadores para inclusão da sustentabilidade na gestão da cadeia de suprimentos vão desde uma reação às pressões externas, como a legislação (Abreu et al., 2004; Dias et al., 2012; Abdala e Barbieri, 2014) até uma maneira proativa de agir que busca adicionar valor ao seu produto e ao seu negócio (Dias et al., 2012; Abdala e Barbieri, 2014).

Neste contexto, inicialmente surgiu a logística reversa como possível solução, a qual há inúmeros conceitos. Carter e Ellram (1998, p. 15) a definiram como o "processo pelo qual as empresas podem se tornar mais eficientes em termos ambientais através da reciclagem, reutilização e redução da quantidade dos materiais utilizados"; segundo o Council of Supply Chain Management Professionals, a logística reversa é um segmento especializado da cadeia de suprimentos com foco no movimento e gestão de produtos e recursos após a venda e após a entrega ao cliente incluindo devoluções de produtos para reparo e/ou crédito (CSCMP, 2005, p. 31); Rogers e Tibben-Lembke (1999, p. 2), adaptando a definição de logística do Council of Supply Chain Management Professionals (antigo Council of Logistics Management), colocam a logística reversa como um "processo de planejar, implementar e controlar o fluxo eficiente e econômico de matérias-primas, estoque em processo, produtos acabados e informações relacionadas do ponto de consumo até o ponto de origem para fins de recapturar ou criar valor ou disposição adequada".

Em seguida, surgiu o conceito de cadeias de suprimento verdes (green supply chain), que, segundo Fetter (2018), foi mencionada pela primeira vez na literatura, em 1996, com Robert B. Handfield observando as melhores práticas na indústria de móveis e com Joseph Sarkis introduzindo um modelo de avaliação sistêmica para práticas comerciais ambientalmente conscientes e estratégicas. Segundo Sarkis (2012) e Miskolcziné (2017), cadeias de suprimento verdes são definidas como a integração de preocupações ambientais no fornecimento e práticas de gerenciamento da cadeia de suprimentos. Também fazem parte dessa cadeia o design de produto, compra e seleção, processos de fabricação, entrega do produto final, uso de produtos e sua eliminação no final da vida útil (Miskolcziné, 2017).

Depois, surgiu o termo "cadeias de suprimento sustentáveis", que tem definição e escopo com uma maior amplitude. Segundo Silva et al. (2015), tomando os preceitos do triple bottom line na gestão da cadeia de suprimentos (em inglês, supply chain management), o conceito de sustainable supply chain management surgiu como uma derivação deste (Linton, 2002; Seuring e Müller, 2008; Beske e Seuring, 2014).

Neste sentido, é importante ressaltar que diversos pesquisadores comprovaram que os modelos de sustentabilidade baseados apenas nessas três dimensões do triple bottom line possuem algumas limitações (Alves et al., 2011; Vizeu et al., 2012; Faria, 2014; Stoffel e Colognese, 2015; Ibarra-Michel, 2018), como, por exemplo, a tentativa de fazer concessões entre os fatores sociais, econômicos e ambientais, como se fossem equivalentes ou intercambiáveis e pudessem ser tratados uns independentes dos outros. 
Quando, na verdade, são facetas interrelacionadas de uma mesma realidade integral. Outros autores, como Wang et al. (2016), Dossou e Nachidi (2017), Morais (2018), Trochu et al. (2018), também apontam que a dimensão social frequentemente tem ficado ausente dentro dos modelos baseados no triple bottom line e o próprio autor do conceito afirmou que é necessário rever este modelo para o futuro capitalismo, estimular a regeneração das economias, sociedades e biosfera e para que haja a criação de valor (Elkington, 2020).

A gestão da cadeia de suprimentos sustentável é conceituada como "a gestão do capital, fluxos, materiais e informações, bem como da cooperação entre empresas ao longo da cadeia de suprimentos, buscando atingir um equilíbrio nas três dimensões: econômica, ambiental e social, que são requisitos de clientes e partes interessadas" (Seuring e Müller, 2008, p. 1700). Isso significa que a gestão da cadeia de suprimentos sustentável pode ser definida como um pensamento estratégico, transparente e integrado para atingir objetivos econômicos, sociais e ambientais numa coordenação sistêmica de processos interorganizacionais ao longo da cadeia (Srivastava, 2007; Seuring e Muller, 2008).

A diferença entre "cadeia de suprimentos verde" e "cadeia de suprimentos sustentável" está na abrangência. Enquanto a "cadeia de suprimentos verde" foca em utilizar produtos e serviços que diminuam os impactos na saúde e no meio ambiente, a "cadeia de suprimentos sustentável" procura reduzir esses impactos e olhar outros aspectos da sustentabilidade como a questão social (Fahimnia et al., 2015; Fetter, 2017).

Neste sentido, pode-se afirmar que as "cadeias de suprimento verdes" têm efeitos imediatos de produtos e serviços no meio ambiente, mas não atendem a sustentabilidade já que ela é um termo mais amplo que aborda as consequências do uso a longo prazo, considera mais aspectos dos impactos sociais e ambientais e aborda outras dimensões como a cultural. Logo, não basta uma cadeia de suprimentos verde, é preciso uma cadeia de suprimentos sustentável.

Independente disso, é importante ressaltar que, no geral, a operação de cadeias de suprimentos verdes ou sustentáveis só pode ser bem sucedida se, além de processos coordenados, os membros da cadeia têm os mesmos objetivos e se concentram no que diz respeito aos clientes (Fetter, 2018). Para isso, segundo Fetter (2018), é necessário uma integração estratégica entre os participantes membros da cadeia de abastecimento.

Segundo Tseng et al. (2019), existem poucos estudos analisando a interseção da "cadeia de suprimentos verde" com a "cadeia de suprimentos sustentável". Ren et al. (2020), afirmam que os estudos existentes estão desatualizados e incompletos e, portanto, são incapazes de fornecer uma análise abrangente da expansão da pesquisa em gestão de suprimentos verdes e sustentáveis. Apesar disso, não se pode negar que houve avanços. Contudo, ainda se está longe de enfrentar os desafios colocados pela complexidade da cooperação interna e incertezas dos mercados externos (Ren et al., 2020).

Especificamente sobre modelos de gestão focados na sustentabilidade, Van Bellen (2004) expôs como lacuna a mensuração por meio de indicadores desenvolvida por razões específicas e restritas impedindo que os mesmos possam ser considerados indicadores de sustentabilidade no seu sentido mais amplo. Munck et al. (2017) apontaram como lacunas: visão da sustentabilidade organizacional como algo meramente instrumental, conflitos de interesses, falta de operacionalização da sustentabilidade, abordagem das dimensões social e ambiental como áreas funcionais da organização, falta de integração das dimensões da sustentabilidade com as competências organizacionais e de interação entre o ambiente externo e a organização.

Esse estudo busca conhecer e avaliar as produções de pesquisas com o tema: cadeias de suprimentos verdes e/ou sustentáveis, no período de 1982 a 2019, identificando os principais autores e documentos, bem como os países e periódicos que mais promovem estudos com essas temáticas.

Para isso, foi usado a análise bibliométrica que foi realizada por meio de indicadores baseados em contagem de número de citações, cocitações, acoplamento e 
outros que possam comprovar a originalidade e a importância do tema na atualidade; e determinar parâmetros como principais autores e artigos publicados.

Espera-se, ao final deste trabalho, demonstrar o estado da arte das cadeias de suprimentos verdes e/ou sustentáveis no período de 1982 a 2019 e, assim, entender a origem e a evolução dos temas, a importância que se tem dado aos mesmos ao longo dos trinta e sete anos analisados, e as áreas do conhecimento e os países que mais têm investido nesses estudos.

\section{Método}

Considerando a necessidade de se compreender o espectro de trabalhos acadêmicos existentes e que tratam, em alguma medida deste tema, foi realizada uma coleta de material, por meio de busca por trabalhos publicados na base de dados Scopus (Elsevier). A Tabela 1 apresenta os atributos e o retorno da busca sobre esse tema.

Tabela 1. Busca por artigos científicos sobre customer centricity.

\begin{tabular}{|l|l|}
\hline Atributo & Descrição Scopus \\
\hline Data da busca: & $12 / 02 / 2020$ \\
\hline Período: & Superior a 1945 \\
\hline $\begin{array}{l}\text { Campos de } \\
\text { pesquisa: }\end{array}$ & Título, Resumo e Palavras-chave \\
\hline Tipo de Publicação: & Todos \\
\hline Chave de Busca: & $\begin{array}{l}\text { (TITLE-ABS-KEY ( "reverse logistics" ) ) AND ( ( TITLE-ABS-KEY ( "green } \\
\text { supply chain")) OR ( TITLE-ABS-KEY ( "sustainable supply chain") ) ) }\end{array}$ \\
\hline Retorno & 244 documentos \\
\hline
\end{tabular}

Fonte: Baseada nos resultados do Scopus (Elsevier).

Mongeon e Paul-Hus (2016) evidenciam que a base de dados Scopus oferece uma cobertura mais abrangente de fontes do que a Web of Science para o campos das Ciências Sociais. Por essas razões, o repositório de dados acadêmicos considerado neste trabalho foi a Scopus (Elsevier).

Nesse sentido, a coleta de dados foi realizada considerando-se a busca por trabalhos que continham o descritor "Reverse Logistics" associado ao termo "Green Supply Chain" ou "Sustainable Supply Chain" no título, resumo ou palavras-chave. Tal busca retornou um conjunto de informações bibliográficas que descrevem as características de 244 trabalhos, revelando um interesse da comunidade acadêmica pelo tema.

Inicialmente, estatísticas descritivas foram usadas para realizar análises de tendências relacionadas à quantidade, área de conhecimento, distribuição geográfica e principais autores relacionados com essa coleção de artigos. Nesse sentido, a análise bibliométrica foi complementada com análises realizadas com base no software VOSviewer para elaborar mapas bibliométricos.

Segundo Chang e Hsieh (2008) e Merigó e Yang (2017), o VOSviewer é útil para visualizar os resultados bibliométricos usando indicadores métricos e sua escolha se deu por ser um software livre, de fácil utilização e capaz de usar os dados Scopus diretamente para gerar visualização de rede (Chang e Hsieh, 2008; Laengle et al., 2017; Merigó e Yang, 2017).

Usando o VOSviewer foi possível fazer a análise relacional de citação pelos métodos de cocitação e de acoplamento bibliográfico. Este tipo de análise permite conhecer as relações estruturais de conectividade teórico-metodológica de um domínio, a 
proximidade, a vizinhança, a associação e a interlocução estabelecida entre documentos e pesquisadores, entre outros, como reconhecidos pela comunidade científica (Grácio, 2016). Por isso, este tipo de análise tem uma abordagem que se caracteriza pela natureza social, histórica e dinâmica e a sua estreita dependência da literatura acadêmica (Hjørland, 2013).

A análise de cocitação mede a relação entre dois ou mais artigos com base no número de publicações em que estes aparecem citados concomitantemente e o acoplamento bibliográfico mensura a proximidade entre dois artigos comparando suas referências e quanto maior o número de referências que compartilham, maior a similaridade entre eles, que pode ser temática, teórico, metodológica ou outra particularidade compartilhada (Lucas et al., 2013), ou seja, o grau de sobreposição da identidade de citação desses autores (Hjørland, 2013). Portanto, pode-se dizer que o acoplamento bibliográfico e a análise de cocitação, como indicadores de similaridade temática, fornecem padrões significativamente diferentes em relação à estrutura de um domínio científico (Small, 1973).

\section{Resultados}

Os 244 trabalhos indexados pela Scopus nos últimos anos representam uma base de conhecimento grande e em rápido crescimento referente ao tema cadeia de suprimentos verdes e sustentáveis. A Figura 2 ilustra a série temporal da publicação desses artigos. É possível verificar que representa um fenômeno que vem sendo estudado recentemente com considerada intensidade, notadamente a partir do ano de 2007, saindo do patamar médio de menos de uma dezena para mais de três dezenas de artigos publicados por ano.

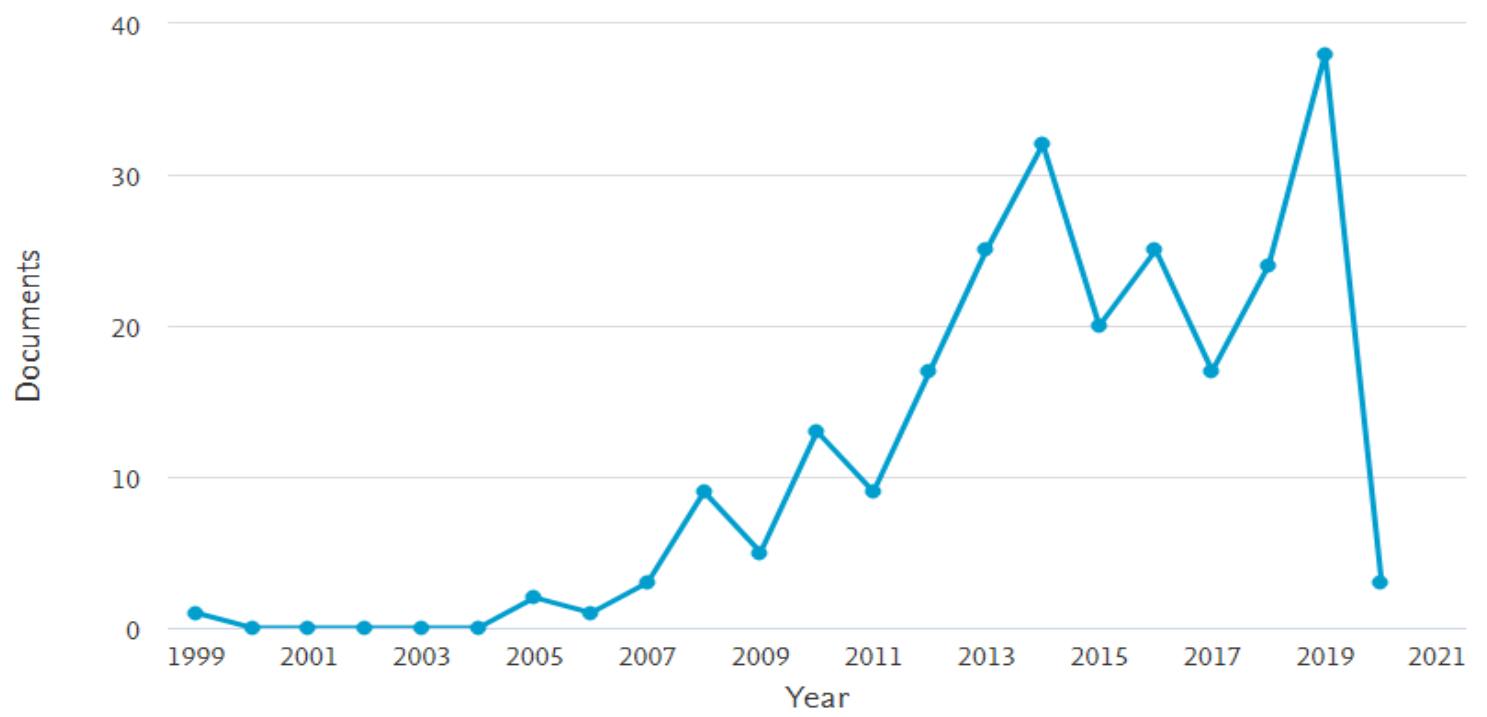

Figura 2. Documentos por ano. Fonte: Scopus, 2020.

Dentre os cinco picos de crescimento, destacam-se dois. 0 ocorrido em 2014 em que se atingiu a produção de 32 documentos e o pico de 2019 quando foram produzidos 38 documentos. 
Verificando estes crescimentos pelos periódicos existentes, foi observado que os periódicos Journal of Cleaner Production e Journal of Production Economics apresentam crescimentos significativos nesta temática desde 2018, conforme Figura 3.

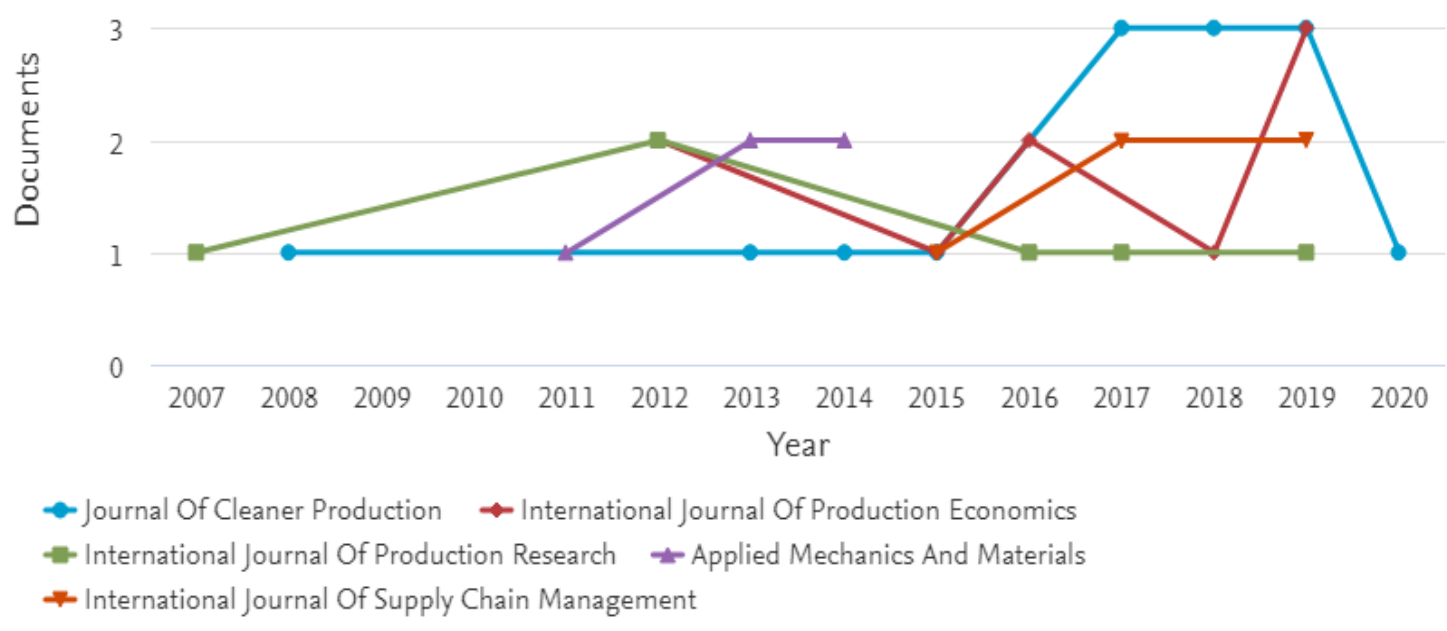

Figura 3. Documentos por periódicos. Fonte: Scopus, 2020.

Observando as áreas do conhecimento, nota-se que o tema está mais presente em 4 áreas: Engenharias, Negócios e gestão, Ciências das Decisões e Ciências da Computação, conforme Figura 4.

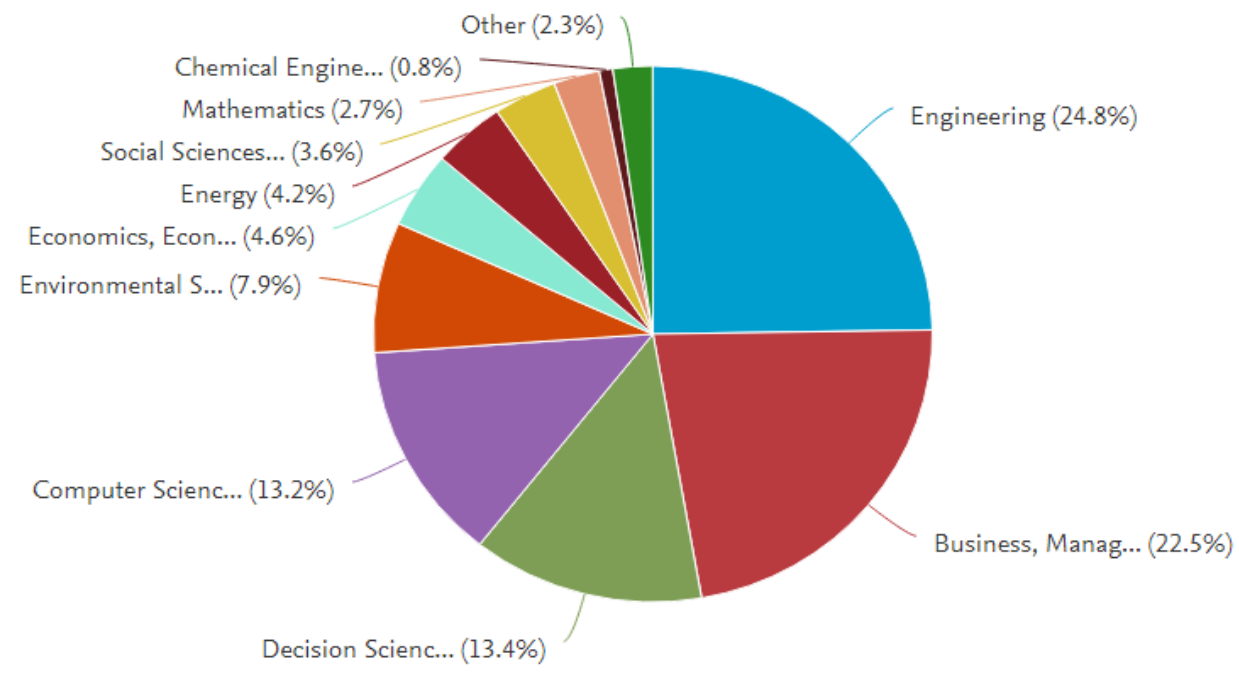

Figura 4. Documentos por área de atuação. Fonte: Scopus, 2020.

Os 244 trabalhos estão distribuídos principalmente nos continentes da Ásia e da América do Norte. Contudo, foi observado produções em diversos países pertencentes a outros continentes, confirmando o interesse mundial no tema. A Figura 5 apresenta o 
ranking com os 10 países que mais publicaram sobre o tema. A Índia é o país que se destaca como a fonte do maior número de publicações, acumulando mais de 35 artigos. Malásia e Estados Unidos vêm em seguida, cada qual com mais de 25 artigos publicados sobre o tema. Considerando esses 10 países, é possível verificar que essa temática está concentrada principalmente em trabalhos publicados na Índia, Malásia, Estados Unidos e China.

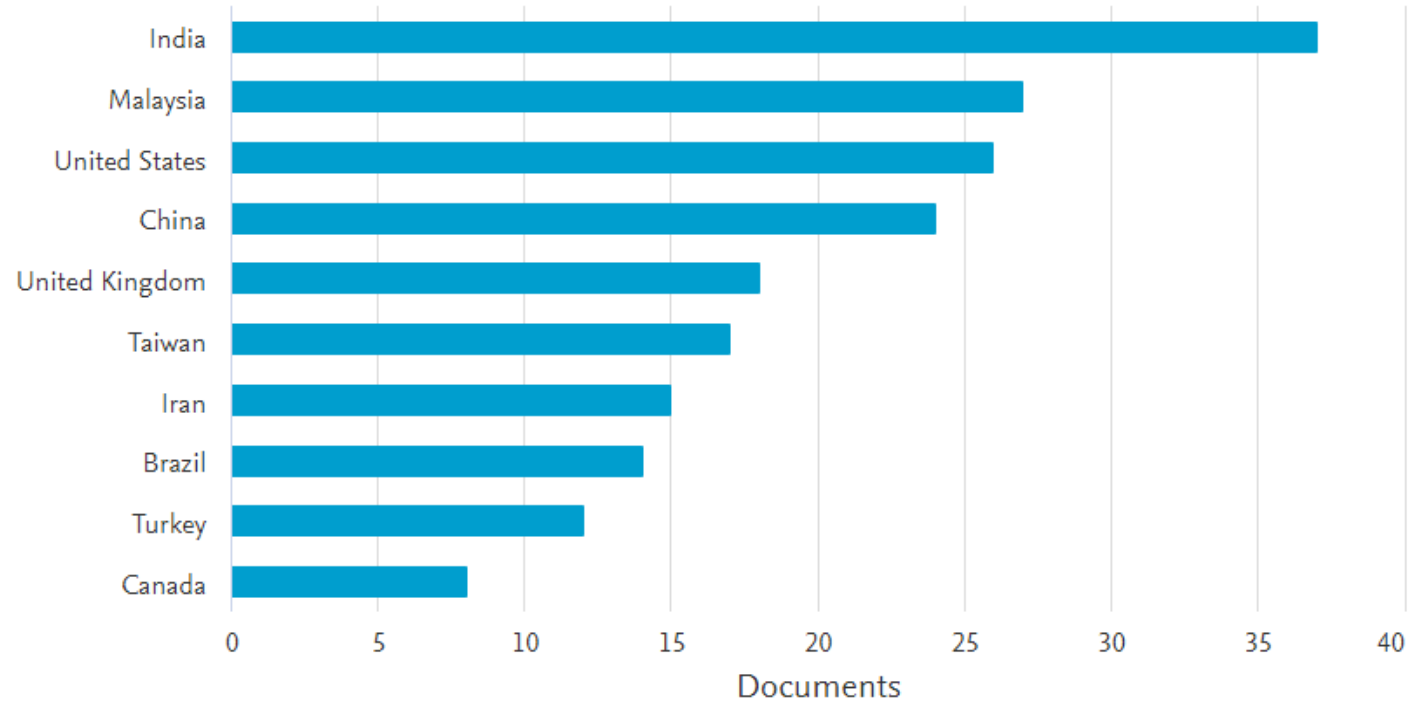

Figura 5. Documentos por região. Fonte: Scopus, 2020.

Entre os autores que mais publicaram sobre o tema, destacam-se aqueles que tradicionalmente produzem trabalhos nesta temática em um campo híbrido entre as áreas de Business e Engenharia. Os 10 autores que formam o topo dessa lista estão demonstrados na Figura 6. A maioria deles atua como professor de Gerenciamento de Operações em universidades da Inglaterra e Estados Unidos como por exemplo Garza-Reyes que leciona na Universidade de Derby e Hazen na Universidade de Ciências Aplicadas da Áustria.

Esses 10 autores são responsáveis pela publicação de 36 artigos que representam cerca de 14,8\% do total de artigos analisados (Figura 2).

Os 10 artigos publicados mais citados e dispostos por ordem decrescente do número de citações foram descritos na Tabela 2.

Pode-se observar que seis dos 10 artigos referem-se a cadeias verdes. Em relação ao demais, dois artigos focam em cadeias de suprimentos sustentáveis, 1 em logística reversa e outro em cadeia de suprimentos fechada.

Em seguida, foram realizadas análises bibliométricas por meio do software VOSviewer (Van Eck e Waltman, 2014). A análise de coocorrência de palavras-chave permite identificar os termos nas pesquisas sobre Cadeias Verdes e Sustentáveis de Logística Reversa que ocorreram com maior frequência. 0 número de coocorrências de dois termos é o número de publicações nas quais os dois termos ocorrem juntas no título, no resumo ou na relação de palavras-chave (Van Eck e Waltman, 2014). 


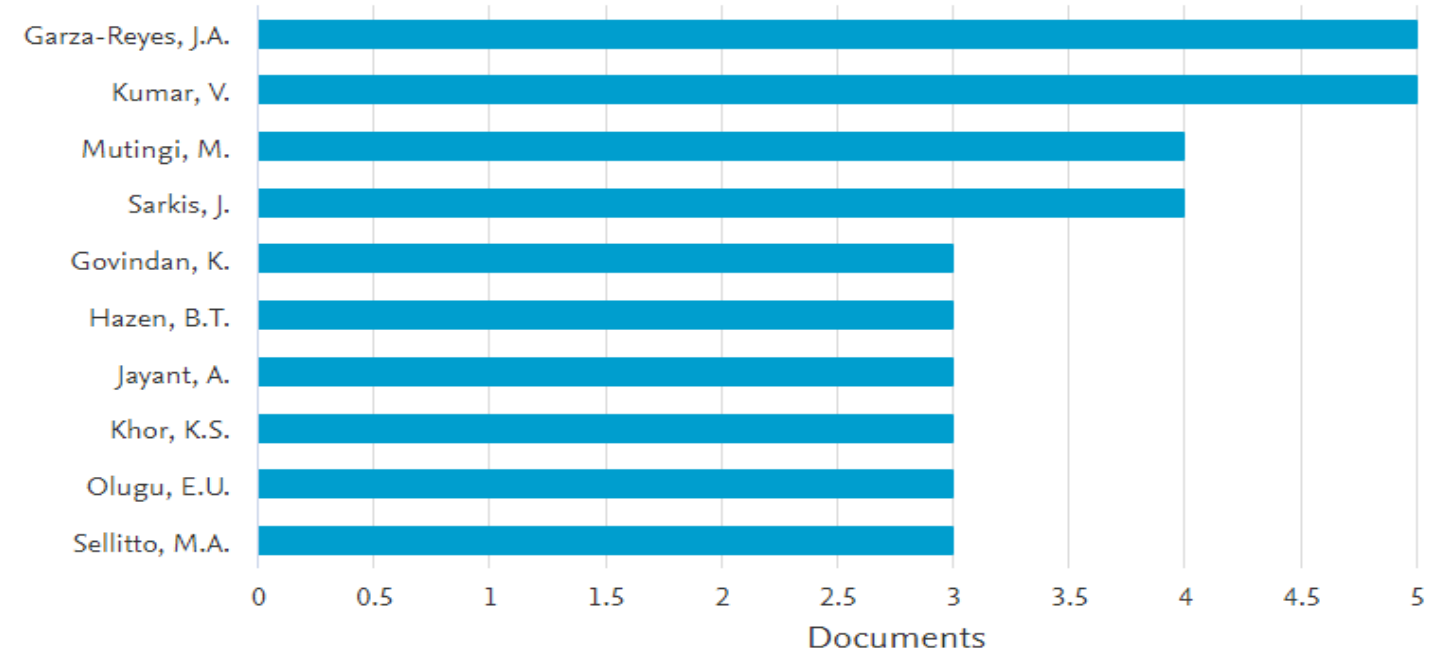

Figura 6. Documentos por autor. Fonte: Scopus, 2020.

Tabela 2. Documentos mais citados.

\begin{tabular}{|l|l|c|c|}
\hline Artigo & Autor & Ano & Citações \\
\hline $\begin{array}{l}\text { Green supply-chain management: A state-of-the- } \\
\text { art literature review }\end{array}$ & Srivastava & 2007 & 1832 \\
\hline $\begin{array}{l}\text { Quantitative models for sustainable supply chain } \\
\text { management: Developments and directions }\end{array}$ & Brandenburg et al. & 2014 & 532 \\
\hline $\begin{array}{l}\text { Design of sustainable supply chains under the } \\
\text { emission trading scheme }\end{array}$ & Chaabane et al. & 2012 & 460 \\
\hline Network design for reverse logistics & Srivastava & 2008 & 351 \\
\hline $\begin{array}{l}\text { An integrated logistics operational model for } \\
\text { green-supply chain management }\end{array}$ & Sheu et al. & 2005 & 347 \\
\hline $\begin{array}{l}\text { Green supply chain initiatives among certified } \\
\text { companies in Malaysia and environmental } \\
\text { sustainability: Investigating the outcomes }\end{array}$ & Eltayeb et al. & 2011 & 311 \\
\hline From reversed logistics to green supply chains & Van Hoek & 1999 & 293 \\
\hline $\begin{array}{l}\text { Environmental principles applicable to green } \\
\text { supplier evaluation by using multi-objective } \\
\text { decision analysis }\end{array}$ & Lu et al. & 2007 & 243 \\
\hline $\begin{array}{l}\text { A closed-loop logistic model with a spanning-tree } \\
\text { based genetic algorithm }\end{array}$ & Wang e Hsu & 2010 & 185 \\
\hline $\begin{array}{l}\text { Development of key performance measures for } \\
\text { the automobile green supply chain }\end{array}$ & Olugu et al. & 2011 & 180 \\
\hline
\end{tabular}

Fonte: Scopus, 2020.

Em relação à coocorrência de termos presentes nos 244 trabalhos selecionados, dos 1.339 termos identificados nos títulos, resumos e palavras-chave, os 9 termos mais citados apareceram com uma frequência mínima de 31 vezes e encontram-se representados na Figura 7. 


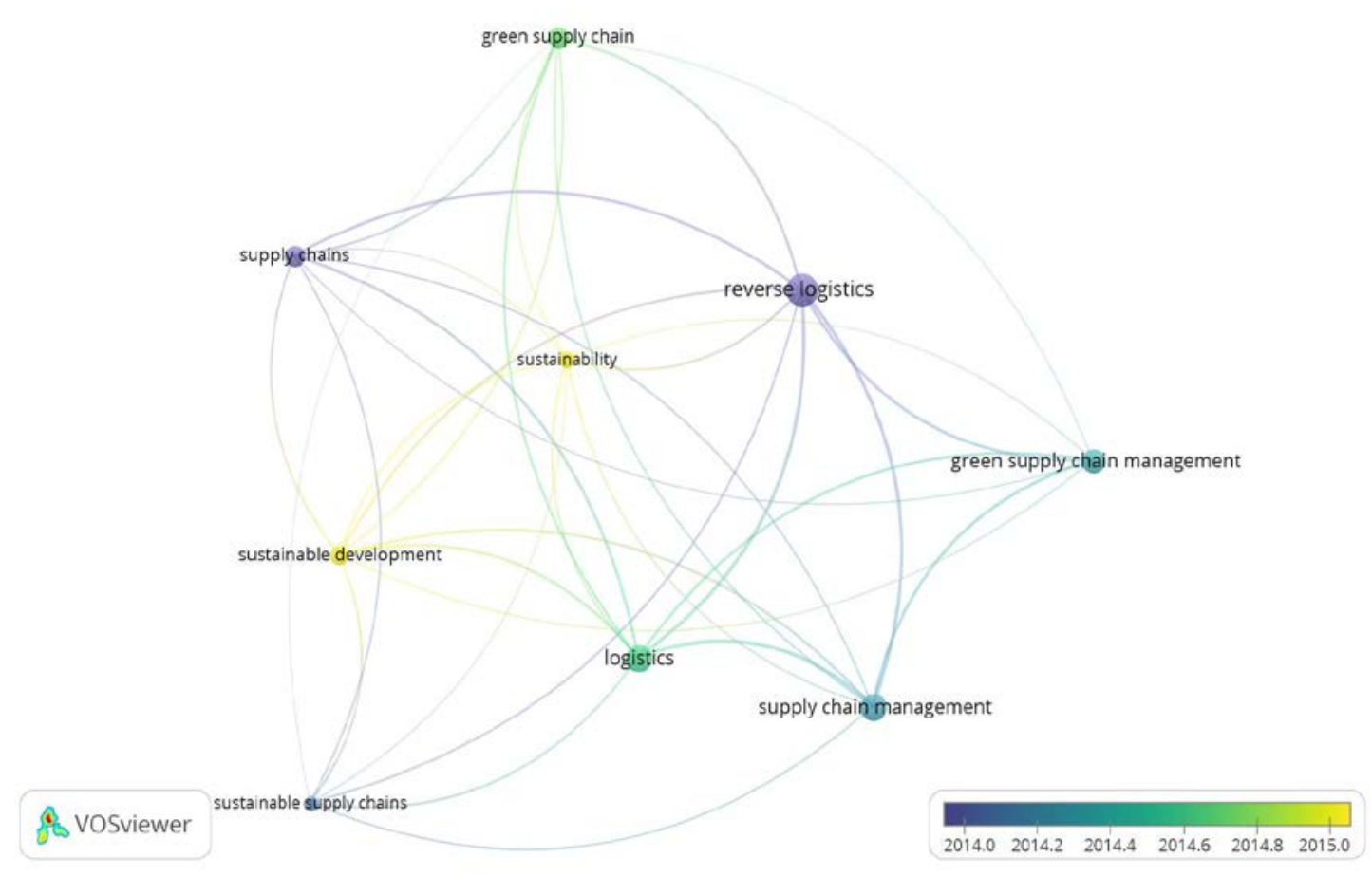

Figura 7. Citações palavras chave. Fonte: VOSviewer, 2020.

Observando a Figura 7, verifica-se que as palavras-chave de destaque são recentes, iniciadas em 2014 e voltadas para: cadeia de suprimentos e logística reversa. Depois começou a se abordar a gestão tanto de cadeia de suprimento quanto de cadeia de suprimento verde. Nos últimos anos, as palavras-chave mais utilizadas são desenvolvimento sustentável e sustentabilidade. Dessa forma, é importante ainda verificar que o termo "green" antecedeu o termo "sustainability".

Avançando com a análise bibliométrica, a análise de cocitação de referência (White e McCain, 1998) foi empregada para analisar a estrutura intelectual dos trabalhos selecionados. A análise de cocitação de referência calcula o número de vezes que dois trabalhos foram citados juntos nas referências dos 244 trabalhos (Zupic e Čater, 2015). Ao se ter em conta que a análise de cocitação considera todas as referências dos documentos selecionados, e não somente as citações elencadas nos artigos de revisão, seus resultados expressam padrões de influência acadêmica tendo como base uma literatura mais ampla (UDOMSAP; HALLINGER, 2020). A Figura 8 apresenta o mapa da rede de cocitações das referências dos artigos considerados (clusters). Dentre as 10.930 referências citadas nos 244 trabalhos, 16 referências atingiram o número mínimo de 8 cocitações. Desta forma, foram identificados 2 clusters. 


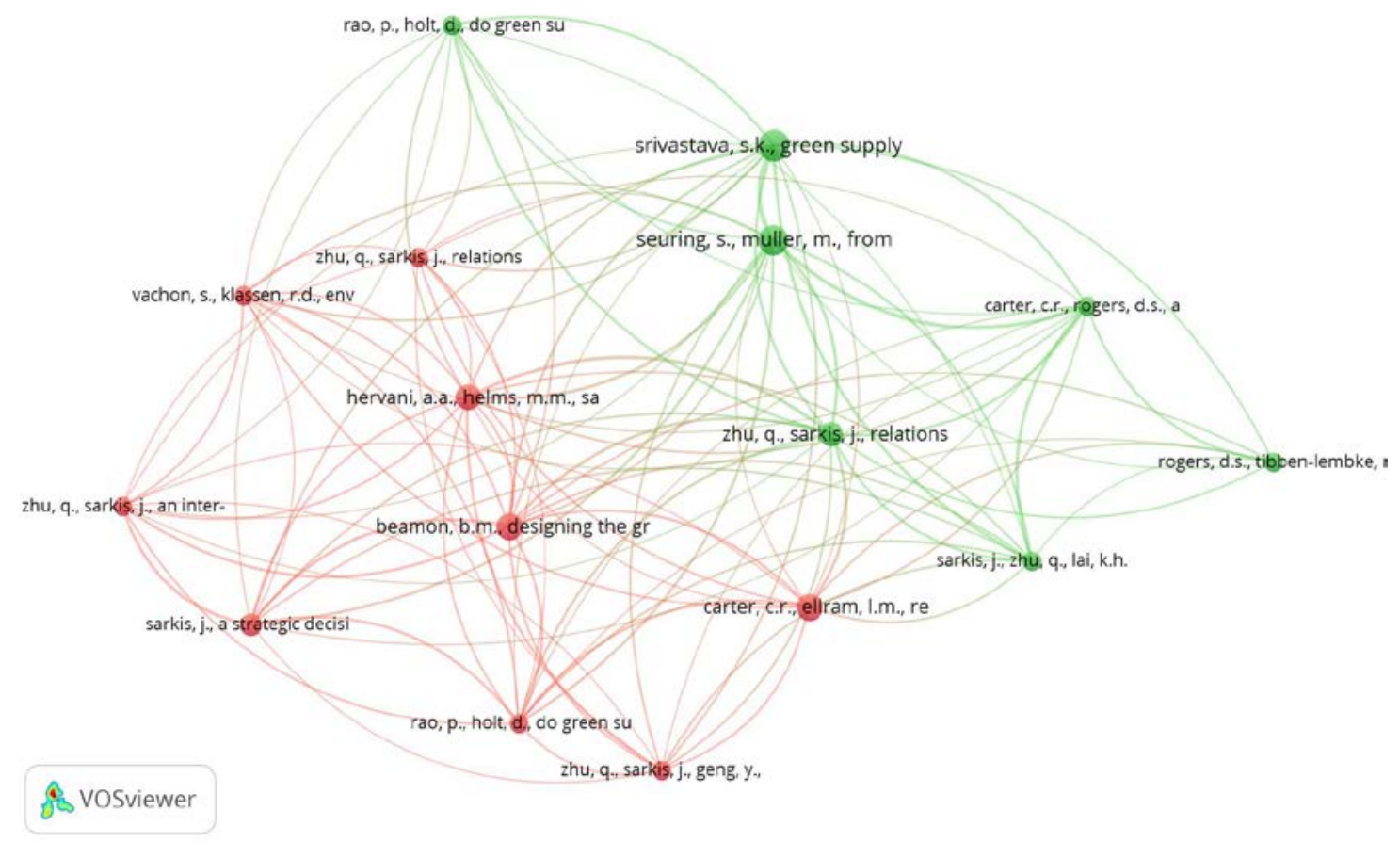

Figura 8. Citações. Fonte: VOSviewer, 2020.

O cluster destacado na cor verde, encabeçado pelos autores: Srivastava (2007) com 19 citações e que aborda a gestão da cadeia de suprimento verde e Seuring e Muller (2008) com 17 citações e que fazem uma revisão da literatura baseada em um framework voltado para a gestão de cadeia de suprimento sustentável. 0 foco deste cluster está na migração de cadeia de suprimentos verde para cadeia de suprimentos sustentável. O outro cluster, em vermelho, é liderado por Beamon (1999) com 14 citações e seu trabalho sobre os formatos das cadeias de suprimento verdes e por Carter e Elram (1998) também com 14 citações e com uma revisão de literatura sobre a logística reversa. Como trabalho mais atual que esses, pode-se citar Rao e Holt (2005) que introduz o conceito de cadeia de suprimentos verde. Reafirmando o identificado anteriormente na análise das palavras chave, este cluster formado por obras mais antigas que o outro, tem como foco a logística reversa e o surgimento da cadeia de suprimentos verdes.

Por fim, o acoplamento bibliográfico usa o número de referências compartilhadas por dois documentos como uma medida da semelhança entre eles. Quanto mais as bibliografias de dois artigos se sobrepõem, mais forte é a conexão (Zupic e Čater, 2015). Para formar o mapa de acoplamento bibliográfico, foram considerados, por meio do software VOSviewer, dentre os 244 trabalhos da base, somente os trabalhos que foram citados por, no mínimo, 100 outros trabalhos científicos. Até o momento da extração da base de dados Scopus. 21 trabalhos atingiram essa marca, conforme apresentado na Figura 9. 


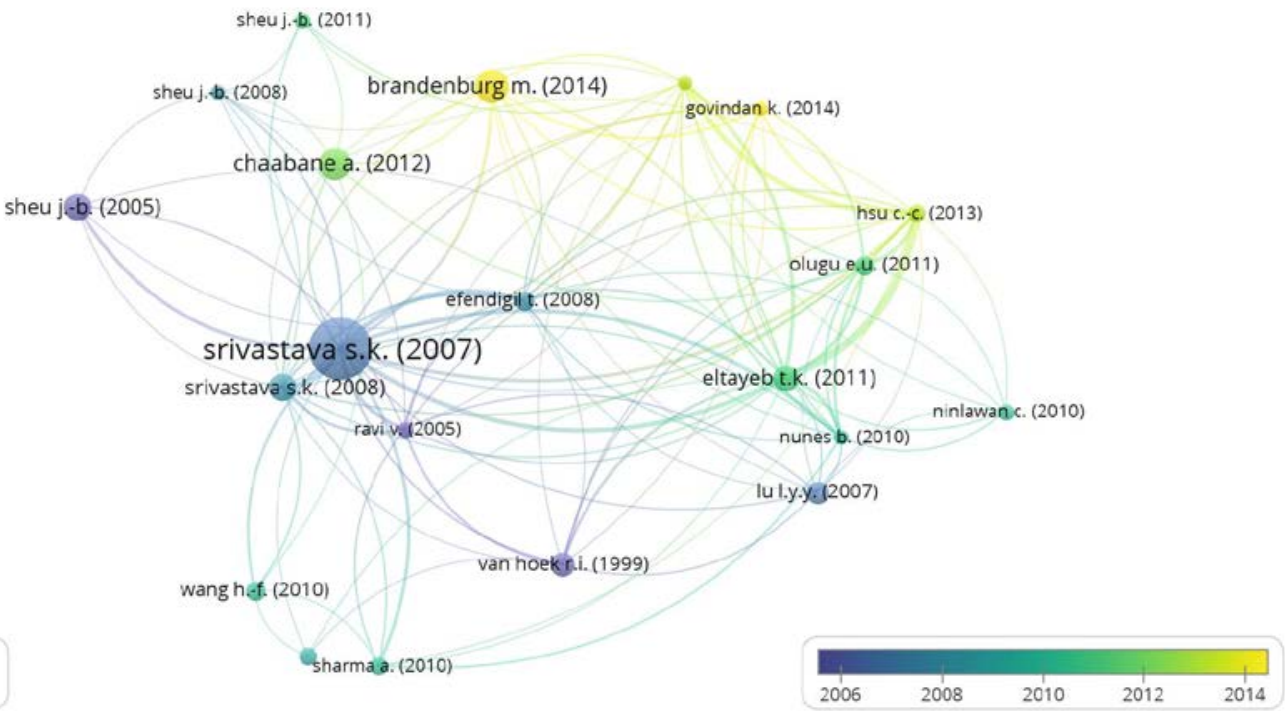

Figura 9. Acoplamento bibliográfico. Fonte: VOSviewer, 2020.

Os artigos com maior acoplamento bibliográfico não necessariamente são os que apresentam a maior quantidade de citações por outros trabalhos, mas sim aqueles que possuem o maior número de referências bibliográficas em comum com os demais artigos considerados na análise. Ao se analisar esses 21 artigos com maior acoplamento bibliográfico, verifica-se que o artigo de Srivastava (2007) que aborda a gestão da cadeia de suprimento verde é o quê mais se destaca dentro deste universo com 1.832 citações.

\section{Conclusões}

As principais limitações desta revisão estão relacionadas à base de dados utilizada (origem dos documentos) que são limitações da própria base de dados Scopus que foi de onde se originou as análises e ao tipo de documento analisado.

Os resultados demonstraram que a inclusão da sustentabilidade na cadeia de suprimentos vai além da chamada cadeia de suprimentos verde. Contudo, o conceito inicial de cadeia de suprimentos sustentável também não significa o alcance real da sustentabilidade já que é baseado no paradigma ultrapassado do triple bottom line. Dessa forma, é necessário mais estudos dentro da cadeia de suprimentos sustentável.

Entretanto, para que esses estudos tenham realmente resultados que caminhem em direção à sustentabilidade da cadeia de suprimentos, é essencial uma definição mais ampla e profunda da sustentabilidade sem, contudo, perder o caráter prático e mensurável. Para isso, é necessário que se insira outras dimensões da sustentabilidade como a cultural e a institucional ao conceito da sustentabilidade e que seja aprofundada as pesquisas nas dimensões do triple bottom line já que, por exemplo, a dimensão social tem sido negligenciada apresentando, assim, várias lacunas.

É relevante destacar que, ao indicar a necessidade de um conceito mais amplo da sustentabilidade, não se está defendendo que todas as organizações adotem o mesmo conceito da sustentabilidade. Entende-se que cada organização deve optar pela abordagem de sustentabilidade que seja mais coerente com seus objetivos, propósitos e estratégias, bem como mais apropriada às circunstâncias sociais nas quais atua e aos valores dominantes que a configura. 
Ante todo o exposto, pode-se afirmar que, hoje, nem a cadeia de suprimentos verde e nem a cadeia de suprimentos sustentável são o que deveriam ser porque não se tem tratado a sustentabilidade em um conceito amplo que transcende a sustentabilidade ambiental, econômica e social. Nesse sentido, pesquisas futuras que elaborem modelos que abordem a gestão das cadeias de suprimentos sustentáveis podem ser muito benéficas.

\section{Conflito de interesses}

Os autores declaram não haver conflito de interesses.

\section{Referências}

Abdala, E. C.; Barbieri, J. C. Determinants of sustainable supply chain: An analysis of mensuration models of pressures and socio-environmental practices. Journal of Operations and Supply Chain Management, v. 7, n. 2, p.110-123, 2014. https://doi.org/10.12660/joscmv7n2p110-123

Abreu, M. C. S.; Rados, G. J. V.; Figueiredo Junior, H. S. As pressões ambientais da estrutura da indústria. RAE - Eletrônica, v. 3, n. 2, Art. 17, 2004. https://doi.org/10.1590/S167656482004000200002

Alves, J. B.; Denardin, V. F.; Silva, C. L. Aproximações entre os principais indicadores de sustentabilidade e as alternativas ao desenvolvimento propostas por E. Leff. Revista de Desenvolvimento Econômico, v. 13, n. 24, 2011.

Batarfi, R.; Jaber, M. Y.; Aljazzar, S. M. A profit maximization for a reverse logistics dualchannel supply chain with a return policy. Computers \& Industrial Engineering, v. 106, p. 58-82, 2017. https://doi.org/10.1016/j.cie.2017.01.024

Beamon, B. M. Measuring supply chain performance. International Journal of $\begin{array}{lllllll}\text { Operations \& Production Management, } & \text { v. 19, } & \text { n. 3, } & \text { p. 275-292, } & 1999 .\end{array}$ https://doi.org/10.1108/01443579910249714

Beske, P.; Land, A.; Seuring, S. Sustainable supply chain management practices and dynamic capabilities in the food industry: A critical analysis of the literature. International Journal of Production Economics, v. 152, p.131-143, 2014. https://doi.org/10.1016/j.ijpe.2013.12.026

Carter, C. R.; Ellram, L. M. Reverse logistics: A review of the literature and framework for future investigation. International Journal of Business Logistics, v. 19, n. 1, p. 85-103, 1998.

Chang, P.-L.; Hsieh, P. Bibliometric overview of operations research: Management Science research in Asia. Asia-Pacific Journal of Operational Research, v. 25, n. 2, p. 217-241, 2008. https://doi.org/10.1142/S0217595908001705

CSCMP - Council and Supply Chain Management Profissional. Supply chain and logistics terms and glossary. 2005. Disponível em: <http://www.cscmp.org/Downloads/ Resorces/glosary03.pdf>. Acesso em: 20 set. 2016.

Dossou, P. E.; Nachidi, M. Modeling supply chain performance. Anais do 27th International Conference on Flexible Automation and Intelligent Manufacturing, Modena, Itália, 2017. 
Elkington, J. 25 years ago I coined the phrase "Triple Bottom Line": Here's why it's time to rethink it. Harvard Business Review, 2020. Disponível em: <https://hbr.org/2018/06/25-years-ago-i-coined-the-phrase-triple-bottom-line-hereswhy-im-giving-up-on-it>. Acesso em: 5 ago. 2021.

Fahimnia, B.; Sarkis, J.; Davarzani, H. Green supply chain management: A review and bibliometric analysis. International Journal of Production Economics, v. 162, p. 101-114, 2015. https://doi.org/10.1016/j.ijpe.2015.01.003

Faria, A. C.; Costa, M. F. G. Gestão de custos logísticos. São Paulo: Atlas, 2012.

Fetter, B. A comprehensive literature review of green supply chain management. Budapest: Budapest University of Technology and Economics, 2018.

Giri, B.; Chakraborty, A.; Maiti, T. Pricing and return product collection decisions in a closed-loop supply chain with dual-channel in both forward and reverse logistics. Journal of Manufacturing Systems, v. 42, p.104-123, 2017. https://doi.org/10.1016/ j.jmsy.2016.11.007

Govindan, K.; Soleimani, H. A review of reverse logistics and closed-loop supply chain: A Journal of Cleaner Production focus. Journal of Cleaner Production, v. 142, Part 1, 2017. https://doi.org/10.1016/j.jclepro.2016.03.126

Grácio, M. C. C. A coplamento bibliográfico e análise de cocitação: revisão teóricoconceitual. Revista eletrônica de biblioteconomia e ciência da informação, v. 21, n. 47, p. 82-99, 2016. https://doi.org/10.5007/1518-2924.2016v21n47p82

Guide, V. D. R.; Harrison, T. P.; Van Wassenhove, L. N. The challenge of closed-loop supply chains. Interfaces, v. 33, n. 6, p. 3-6, 2003.

Heydari, J.; Govindan, K.; Jafari, A. Reverse and closed loop supply chain coordination by considering government role. Transportation Research, Part D: Transport and Environment, v. 52, p. 379-398, 2017.

Hjørland, B. Citation analysis: A social and dynamic approach to knowledge organization. Information Processing and Management, v. 49, n. 6, p. 1313-1325, 2013.

Ibarra-Michel, J. P. Dimensión social desde el realismo crítico. Revista Metropolitana de Sustentabilidade, v. 8, n. 3, p. 142-158, 2018.

Kazemi, N.; Modak, N. M.; Govindan, K. A review of reverse logistics and closed loop supply chain management studies published in IJPR: A bibliometric and content analysis. International Journal of Production Research, v. 57, n. 15/16, p. 4937-4960, 2018. https://doi.org/10.1080/00207543.2018.1471244

Lagarinhos, C. A. F.; Tenório, J. A. S.. Logística reversa dos pneus usados no Brasil. Polímeros, v. 23, n. 1, 2013. https://doi.org/10.1590/S0104-14282012005000059

Lee, W.; Wang, S. P.; Chen, W. C. Forward and backward stocking policies for a two-level supply chain with consignment stock agreement and stock-dependent demand. European

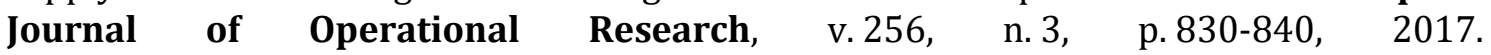
https://doi.org/10.1016/j.ejor.2016.06.060

Lucas, E. O.; Garcia-Zorita, J. C.; Sanz-Casado, E. Evolução histórica de investigação em informetria: ponto de vista espanhol. Liinc em Revista, v. 9, n. 1, p. 255-270, 2013. https://doi.org/10.18617/liinc.v9i1.509 
Laengle, S.; Merigó, J. M.; Miranda, J.; Słowiński, R.; Bomze, I.; Borgonovo, E.; Dyson, R. G.; Oliveira, J. F.; Teunter, R. Forty years of the European Journal of Operational Research: A bibliometric overview. European Journal of Operational Research, v. 262, n. 3, p. 803-816, 2017. https://doi.org/10.1016/j.ejor.2017.04.027

Linton, J. D.; Yeomans, J. S.; Yoogalingam, R. Supply planning for industrial ecology and remanufacturing under uncertainty: A numerical study of leaded-waste recovery from television disposal. Journal of the Operational Research Society, v. 53, n. 11, p. 1185-1196, 2002. https://doi.org/10.1057/palgrave.jors.2601418

Merigó, J. M.; Yang, J. B. A bibliometric analysis of operations research and management science. Omega, v. 73, p. 37-48, 2017. https://doi.org/10.1016/j.omega.2016.12.004

Mongeon, P.; Paul-Hus, A. The journal coverage of Web of Science and Scopus: A comparative analysis. Scientometrics, v. 106, n. 12, p. 213-228, 2016. https://doi.org/10.1007/s11192-015-1765-5

Morais, D. O. C. 0 pilar social: a dimensão invisível na gestão da cadeia de suprimentos sustentável. São Paulo: FGV EAESP CDAE, 2018. (Tese de doutorado).

Munck, L.; Bansi, A. C.; Galleli, B. Sustentabilidade em contexto organizacional: uma análise comparativa de modelos que propõem trajetórias para sua gestão. Revista de Ciências da Administração, $\quad$ v. 18, $\quad$ n. 44, p. 91-110, 2016. https://doi.org/10.5007/21758077.2016v18n44p91

Pedram, A.; Yusoff, N. B.; Udoncy, O. E.; Mahat, A. B.; Pedram, P.; Babalola, A. Integrated forward and reverse supply chain: A tire case study. Waste Management, v. 60, p. 460-470, 2017. https://doi.org/10.1016/j.wasman.2016.06.029

Rao, P.; Holt, D. Do green supply chains lead to competitiveness and economic performance? International Journal of Operations \& Production Management, v. 25, n.9, p. 898-916, 2005. https://doi.org/10.1108/01443570510613956

Ren, R.; Hu, W.; Dong, J.; Sun, B.; Chen, Y.; Chen, Z. A systematic literature review of green and sustainable logistics: Bibliometric analysis, research trend and knowledge taxonomy. International Journal Environmental Research and Public Health, v. 17, n. 1, 2020. https://doi.org/10.3390/ijerph17010261

Rogers, D. S.; Tibben-Lembke, R. S. Going backwards: Reverse logistics trends and practices. Reno, University of Nevada, 1999.

Sarkis, J.; Zhu, Q. H.; Lai, K. An organizational theoretic review of green supply chain management literature, International Journal of Production Economics, v. 130, n. 1, p. 1-15, 2011. https://doi.org/10.1016/j.ijpe.2010.11.010

Seuring, S.; Müller, M. From a literature review to a conceptual framework for sustainable supply chain management. Journal of Cleaner Production, v. 16, n. 15, p. 1699-1710, 2008. https://doi.org/10.1016/j.jclepro.2008.04.020

Silva, M. E.; Neutzling, D. M.; Alves, A. P. F.; Dias, P.; Santos, C. A. F.; Nascimento, L. F. Sustainable supply chain management: A literature review on Brazilian. Journal of Operations and Supply Chain Management, v. 8, n. 1, p. 29-45, 2015. https://doi.org/10.12660/joscmv8n1p29-45

Small, H. Cocitation in the scientific literature: A new measure of the relationship between two documents. Journal of the American Society for Information Science, v. 24, n. 4 , p. 265-269, 1973. https://doi.org/10.1002/asi.4630240406 
Srivastava, S. K. Green supply-chain management: A state-of-the-art literature review. International Journal of Management Reviews, v. 9, n. 1, p. 53-80, 2007. https://doi.org/10.1111/j.1468-2370.2007.00202.x

Stoffel, J. A.; Colognese, S. A. O desenvolvimento sustentável sob a ótica da sustentabilidade multidimensional. Revista da FAE, v. 18, n. 2, 2015.

Trochu, J.; Chaabane, A.; Ouhimmou, M. Reverse logistics network redesign under uncertainty for wood waste in the CRD industry. Resources, Conservation \& Recycling, v. 128, p. 32-47, 2018. https://doi.org/10.1016/j.resconrec.2017.09.011

Tseng, M.-L.; Islam, Md. S.; Karia, N.; Fauzi, F. A.; Afrin, S. A literature review on green supply chain management: Trends and future challenges. Resources, Conservation \& Recycling, v. 141, p. 145-162, 2019. https://doi.org/10.1016/j.resconrec.2018.10.009

Udomsap, A. D.; Hallinger, P. A bibliometric review of research on sustainable construction 1994-2018. Journal of Cleaner Production, v. 254, 2020. https://doi.org/10.1016/ j.jclepro.2020.120073

Van Bellen, H. M. Indicadores de sustentabilidade: um levantamento dos principais sistemas de avaliação. Cadernos EBAPE.BR, v. 2, n. 1, 2004. https://doi.org/10.1590/ S1679-39512004000100002

Van Eck, N. J.; Waltman, L. Visualizing bibliometric networks. In: Ding, Y.; Rousseau, R.; Wolfram, D. (Eds.). Measuring scholarly impact: Methods and practice. London: Springer, 2014. p. 285-320.

Vizeu, F.; Meneghetti, F. K.; Seifert, R. E. Por uma crítica ao conceito de desenvolvimento sustentável. Cadernos EBAPE.BR, v. 10, n. 3, art. 6, 2012. https://doi.org/10.1590/S167939512012000300007

Wang, J.-J.; Chen, H.; Rogers, D. S.; Ellram, L. M.; Grawe, S. J.; Ellinger, A. E. A bibliometric analysis of reverse logistics research (1992-2015) and opportunities for future research. International Journal of Physical Distribution \& Logistics Management, v. 47, n. 8, p. 666-687, 2016. https://doi.org/10.1108/ijpdlm-10-2016-0299

White, H. D.; McCain, K. W. Visualizing a discipline: An author co-citation analysis of information science 1972-1995. Journal of the American Society for Information Science, v 49, n. 4, p. 327-355, 1998. https://doi.org/10.1002/(SICI)10974571(19980401)49:4<327::AID-ASI4>3.0.CO;2-4

Zheng, B.; Yang, C.; Yang, J.; Zhang, M. Pricing, collecting and contract design in a reverse supply chain with incomplete information. Computers and Industrial Engineering, v. 111, Art. C, p. 109-122, 2017. https://doi.org/10.1016/j.cie.2017.07.004

Zhu, Q.; Sarkis, J. Relationships between operational practices and performance among early adopters of green supply chain management practices in chinese manufacturing enterprises. Journal of Operations Management, v. 22, n. 3, p. 265-289, 2004. https://doi.org/10.1016/j.jom.2004.01.005

Zupic, I.; Čater, T. Bibliometric methods in management and organization. Organizational Research Methods, v. 18, n. 3, p. 429-472, 2015. https://doi.org/10.1177/ 1094428114562629

Informação da Licença: Este é um artigo Open Access distribuído sob os termos da Licença Creative Commons Attribution, que permite uso irrestrito, distribuição e reprodução em qualquer meio, desde que a obra original seja devidamente citada. 